\title{
CALIDAD DE VIDA Y PRESTACIONES EN SALUD EN PACIENTES CON DIABETES MELLITUS TIPO 2 SEGÚN REGIÓN GEOGRÁFICA EN ARGENTINA
}

\author{
QUALITY OF LIFE AND HEALTH BENEFITS IN PATIENTS WITH TYPE 2 \\ DIABETES ACCORDING TO GEOGRAPHY REGION IN ARGENTINA
}

\begin{abstract}
María Amelia Linari', Claudio González², Guillermo Dieuzeide³, María Florencia Badia ${ }^{4}$, María Inés Argerich ${ }^{5}$, Mariela Echenique ${ }^{6}$, Gustavo Frechtel7 , Omar Álvaro ${ }^{6}$, María del Carmen Babus $^{8}$, Leticia Barrera ${ }^{9}$, Natalia Blanco ${ }^{10}$, Diego Botta ${ }^{11}$, María Marta Curet ${ }^{12}$, Raúl David ${ }^{5}$, Alejandra Dib ${ }^{13}$, Daniel Dionisi ${ }^{10}$, Susana Fuentes ${ }^{14}$, Sandra Geraci ${ }^{15}$, Natalia Laguarde ${ }^{13}$, Silvia Lapertosa ${ }^{16}$, Astrid Libman ${ }^{17}$, Elizabeth Méndez ${ }^{10}$, Julieta Méndez ${ }^{18}$, Luis Neira ${ }^{13}$, María Florencia Noguero ${ }^{14}$, Verónica Ojeda ${ }^{19}$, Nelson Papini ${ }^{20}$, Víctor Previtera ${ }^{5}$, Erika Vago ${ }^{21}$, Carla Wassner ${ }^{22}$, Débora Chan ${ }^{23}$
\end{abstract}

\section{RESUMEN}

Introducción: la adherencia al tratamiento de la diabetes mellitus tipo 2 (DM2) en el adulto es un tema complejo y multifactorial que aúna aspectos propios de la enfermedad como no propios, los cuales impactan en la calidad de vida. En Argentina se presentan grandes dificultades al costear la totalidad del tratamiento de la enfermedad. El impacto del ingreso sobre el gasto en salud en DM2 es mayor para los niveles más bajos de ingresos.

Objetivos: analizar en adultos con DM2 en Argentina diferencias en variables de calidad de vida y objetivos metabólicos según regiones geográficas y prestación en salud; conocer el gasto de bolsillo en salud y los costos en salud vinculados a DM2; establecer la asociación entre el gasto de bolsillo con variables socioeconómicas y el cumplimiento de los objetivos metabólicos.

Materiales y métodos: sobre ocho regiones geográficas de la República Argentina se incluyeron 1.520 pacientes adultos con DM2 y se los clasificó según prestación en salud (sin prestación, con prestación o jubilados). Se aplicó cuestionario validado autorreferido.

Resultados: se encontraron diferencias significativas por región geográfica para jubilación prematura por DM2, pérdida de percepción de calidad de vida y cumplimiento de los objetivos metabólicos. Se encontró significancia estadística del gasto de bolsillo con la presencia de subsidio familiar, la jubilación prematura por DM2 y el cumplimiento de los objetivos metabólicos.

Conclusiones: se observó disparidad entre las variables de calidad de vida, el gasto en salud y la percepción de subsidios.

Palabras clave: diabetes mellitus tipo 2; adulto; adherencia al tratamiento; calidad de vida; prestación de atención en salud.

Revista de la Sociedad Argentina de Diabetes 2019; Vol. 53 (87-96)

\section{ABSTRACT}

Introduction: adherence to treatment of diabetes mellitus type 2 (DT2) in the adult is a complex and multifactorial issue that combines aspects of the disease as not own, which impact on the quality of life. In Argentina there are great difficulties in paying for the entire treatment of the disease. The impact of income on health spending in DT2 is greater for lower income levels.

Objectives: to analyze differences in quality of life and metabolic objectives according to geographic regions and health benefits in adults with DT2 in Argentina; know the out of pocket expenses in health and health costs linked to DT2; establish an association between out of pocket spending with socioeconomic variables and compliance with metabolic objectives.

Materials and methods: over eight geographic regions of the Argentine Republic, 1.520 adult patients with DT2 were classified according to health benefit (without benefit, with benefits or retirees). Validated self-reported questionnaire was applied. Results: significant differences were found by geographic region for premature retirement due to DT2, the loss of perception of quality of life and the fulfillment of metabolic objectives. Statistical significance of out-of-pocket spending was found with the presence of family allowance, early retirement by DT2 and compliance with metabolic objectives.

Conclusions: disparity between the variables of quality of life, health expenditure and perception of subsidies was observed.

Key words: diabetes mellitus type 2; adult; adherence to treatment; quality of life; provision of health care.

Revista de la Sociedad Argentina de Diabetes 2019; Vol. 53 (87-96) 
1 Coordinación de la Sección Endocrinología y Nutrición, Unión Obrera Metalúrgica (UOM), Vicente López, Provincia de Buenos Aires, Argentina

2 Docente titular, Departamento de Farmacología de la Universidad Nacional de Buenos Aires, Ciudad Autónoma de Buenos Aires, Argentina

3 Jefe del Servicio del Hospital Nuestra Sra. del Carmen de Chacabuco, Provincia de Buenos Aires, Argentina

4 Médica de Planta, Hospital Provincial de Rosario, Santa Fe, Argentina

5 Médica/o de Planta, Hospital Perrupato, San Martín, Mendoza, Argentina

6 Médica/o del staff del Centro Integral de Diabetes, Neuquén

7 Coordinador del Servicio de Nutrición, Hospital Sirio Libanés, Ciudad Autónoma de Buenos Aires, Argentina

8 Médica de Planta, Centro Integral de Diabetes, Endocrinología y Nutrición (CIDEN), Jujuy, Argentina

9 Médica de Planta, Centro Médico Carrodilla y Obra Social de Empleados Públicos (OSEP), Maipú, Mendoza, Argentina

10 Médica/o de Planta, Hospital de Agudos Ramón Madariaga, Misiones, Argentina

11 Médico de Planta, Hospital Bouquet, Neuquén, Argentina

12 Médica de Planta, Hospital Regional Dr. Ramón Carrillo, Santiago del Estero, Argentina

13 Médica/o del Servicio de Nutrición, Hospital Sirio Libanés, Ciudad Autónoma de Buenos Aires, Argentina
14 Médica de Planta, Hospital El Cruce, Provincia de Buenos Aires, Argentina

15 Médica del Servicio de Nutrición, Hospital Castex de San Martín, Provincia de Buenos Aires, Argentina

16 Médica de Planta, Hospital Central J.R. Vidal, Corrientes, Argentina

17 Sanatorio Americano, Rosario, Santa Fe, Argentina

18 Médica de Planta, Hospital Vélez Sarsfield, Ciudad Autónoma de Buenos Aires, Argentina

19 Jefa del Servicio del Hospital Nacional de Clínicas, Córdoba, Argentina

20 Referente de Diabetes en el Área Departamental Luján de Cuyo, Mendoza, Argentina

21 Médica especialista, Centro Privado en Regina, Río Negro, Argentina

22 Médica del Servicio de Nutrición, Hospital Udaondo y Privado, Carapachay, Provincia de Buenos Aires, Argentina

23 Docente en la Universidad Tecnológica (UTN), Facultad Regional Buenos Aires, Ciudad Autónoma de Buenos Aires, Argentina

Contacto de la autora: María Amelia Linari

E-mail: marimelina@yahoo.com

Correspondencia: Liniers 1752 (CP1602), Florida, Provincia

de Buenos Aires, Argentina

Fecha de trabajo recibido: 13/02/19

Fecha de trabajo aceptado: 25/05/19

Conflictos de interés: los autores declaran que no existe conflicto de interés.

\section{INTRODUCCIÓN}

En Argentina, a pesar de la implementación de la Ley N²3.753', su modificación en la Ley $26.914^{2}$ y el Programa Médico Obligatorio, los pacientes con diabetes mellitus tipo 2 (DM2) residentes en nuestro país sin y con prestaciones en salud privada u obras sociales presentan grandes dificultades para costear la totalidad del tratamiento ${ }^{3}$. Si bien en noviembre de 2018 se amplió la cobertura, la mencionada todavía no se aplica en todo el país ${ }^{4}$ y las diferencias en el acceso son significativas.

Los pacientes adultos con DM2 presentan en su mayoría comorbilidades asociadas como la hipertensión arterial, dislipidemias y obesidad que requieren fármacos, seguimiento e intervenciones de otras disciplinas. El aumento de la incidencia de complicaciones crónicas acentúa la necesidad de atención médica, recursos en salud e insumos ${ }^{5,6}$. Paralelo a la desigual accesibilidad a los sistemas de salud existen también diferencias en los ingresos, con inequidad del gasto de bolsillo para la salud respecto del ingreso en los hogares. El gasto en las familias de bajos recursos para la compra de bienes y servicios de salud representa una proporción mayor de sus ingresos de lo que significa en la población con mayores recursos. Aunque el paciente y su familia posean prestaciones o algún sistema de seguro de salud, con frecuencia de- ben realizar desembolsos como copagos. Estas situaciones influyen significativamente sobre las conductas de adherencia al tratamiento7,8.

Los adultos con DM2 tienen más costos en salud, ingresos más bajos y mayores gastos totales fuera de su bolsillo, en comparación con los adultos con otras patologías crónicas como cardiopatías, hipertensión y cáncer ${ }^{9}$. Las mujeres adultas con DM2 que viven en la pobreza y las personas con enfermedades coexistentes tienen mayores probabilidades de altos gastos en salud. Entre los adultos que no son ancianos sin cobertura pública y los no asegurados se muestra mayor riesgo de altas cargas, en comparación con los que poseen seguro. Más del $23 \%$ de las personas sin seguro y más del $20 \%$ con cobertura pública utilizan más de la mitad de sus ingresos para la atención de la salud. La Tercera Encuesta Nacional de Factores de Riesgo informó sobre la mayor prevalencia de DM2 asociada a menos ingresos, menores niveles de educación y a la cobertura en salud de tipo pública ${ }^{10}$.

Las recomendaciones y guías de práctica clínica actuales, que sugieren estándares de gestión en el tratamiento y evaluación de la calidad de atención y adherencia, reflejan la atención subóptima que no cumple con el objetivo estándar e impactan negativamente en la calidad de vida de los pacientes adultos con DM2. La Organización 
Mundial de la Salud (OMS) considera que la falta de adherencia es un tema prioritario en la Salud Pública con fracasos terapéuticos, aumento de la hospitalización y de los costos sanitarios ${ }^{11}$.

La falta de estudios multicéntricos según accesibilidad y región mediante el uso de cuestionarios validados en Argentina conlleva a carencias en resultados propios actuales y deficiencias al momento de intervenir en medidas de Salud Pública. Asimismo la carencia de resultados dificulta la correlación de la situación actual respecto de la adherencia al tratamiento, variables de calidad de vida vs los estándares de objetivos a alcanzar referidos en las recomendaciones y directrices de entidades internacionales ${ }^{12}$.

A raíz de las necesidades sobre el tema adherencia y la situación de salud en Argentina se diseñó, a partir de dos encuestas, un cuestionario sobre adherencia y cumplimiento en el tratamiento. La primera parte -referida a antecedentes personales, costos y motivos de incumplimiento a la prescripción- se constituyó sobre la idea y base de un cuestionario realizado en 2001 y validado en $2010^{13,14}$. La segunda parte de la encuesta se tradujo al castellano y se adaptó de un cuestionario titulado: Summary of Diabetes Self Care Activities (SDSCA), construido y validado psicométricamente ${ }^{15}$. Luego, utilizando el cuestionario en 176 pacientes en 2013, se realizó un estudio de tipo descriptivo ${ }^{12}$. Los resultados encontrados en esos momentos informaron una clara asociación positiva constante entre la dificultad en la adherencia al tratamiento y el aumento del riesgo. En dicho estudio también se observó una asociación negativa entre la cantidad de personas convivientes en el hogar y el porcentaje del presupuesto invertido en la enfermedad, y una asociación positiva entre la proporción del gasto de bolsillo en salud y los respondedores con subsidio o ayuda económica. Se demostró que el impacto del ingreso sobre el gasto en salud era mayor para los que tenían niveles bajos de ingresos, por consiguiente un aumento en el nivel de salud provocó una disminución del gasto de bolsillo mucho más fuerte para aquellos cuyo nivel de ingreso era más bajo ${ }^{16}$. Es necesario en adultos con DM2, según prestación en salud y región geográfica de Argentina, analizar y establecer la dimensión del problema que representa la adherencia al tratamiento, evaluar diferencias observadas y su impacto en la calidad de vida, como también las variables más significativas que impactan en ella.

\section{OBJETIVOS Objetivo principal}

- Analizar en adultos con DM2 en Argentina diferencias en variables de calidad de vida y metas u objetivos metabólicos según regiones geográficas y prestación de atención en salud.

\section{Objetivos específicos}

- Analizar en pacientes adultos con DM2 según región geográfica de Argentina y prestación en salud variables de calidad de vida.

- Evaluar la disparidad en la reducción del sueldo por ausentismo, jubilación prematura, percepción de pérdida de calidad de vida y su asociación con objetivos metabólicos glucémicos.

- Identificar el gasto de bolsillo en salud y los costos en salud vinculados a DM2 de los pacientes y su hogar.

- Establecer asociación entre el gasto de bolsillo con variables socioeconómicas y el cumplimiento de objetivos metabólicos.

\section{MATERIALES Y MÉTODOS \\ Diseño}

Estudio de tipo transversal.

\section{Población}

Pacientes adultos con DM2 que se asistieron en diferentes centros de atención en Argentina.

\section{Muestra}

Se obtuvo de ocho regiones de Argentina que correspondieron a los capítulos de trabajo que divide y trabaja la Sociedad Argentina de Diabetes (SAD). Cada región se propuso reclutar no menos de 65 pacientes con DM2 por cada uno de los tres centros de atención (195 por zona geográfica) públicos y privados. Se incluyeron 1.520 pacientes adultos con diagnóstico de DM2 que presentaban tres meses o más de antigüedad de la enfermedad, previo consentimiento informado firmado. El porcentual de no respuesta para el valor objetivo total correspondió a un 2,56\%. Se agruparon en: adultos mayores de 65 años jubilados, y adultos con y sin prestación en salud (prepaga o mutual u obra social). No se incluyeron pacientes con disglucemia. Las encuestas se respondieron en la sala de espera o en su domicilio y se devolvieron al investigador (Tablas 1 y 2 ). 


\begin{tabular}{|l|c|c|}
\hline \multicolumn{1}{|c|}{ Región } & N & \% \\
\hline Buenos Aires & 261 & 17,17 \\
\hline CABA & 167 & 10,99 \\
\hline Comahue & 224 & 14,74 \\
\hline Centro & 67 & 4,41 \\
\hline Cuyo & 229 & 15,07 \\
\hline Litoral & 188 & 12,37 \\
\hline Noroeste & 140 & 9,21 \\
\hline Noreste & 244 & 16,05 \\
\hline
\end{tabular}

Fuente: elaboración propia.

N: número de encuestados; CABA: Ciudad Autónoma de Buenos Aires.

Tabla 1: Descripción de la muestra de encuestados (N:1.520).

\begin{tabular}{|l|c|c|}
\hline \multicolumn{1}{|c|}{ Cobertura } & N & \% \\
\hline Jubilado & 554 & 36,45 \\
\hline OS & 481 & 31,64 \\
\hline SOS & 485 & 31,91 \\
\hline
\end{tabular}

Fuente: elaboración propia.

N: número de encuestados; OS: con obra social; SOS: sin obra social.

Tabla 2: Distribución de encuestados según cobertura en salud.

\section{Variables}

- Aspectos personales. Incluyeron: edad, sexo, presencia y tipo de prestación de salud (sin obra social, con obra social y jubilación) y fecha de diagnóstico de DM2 ${ }^{12}$.

- Aspectos educacionales. No lee ni escribe, primario incompleto, primario completo, secundario incompleto, secundario completo, terciario o universitario incompleto, terciario o universitario completo. Para medir asociaciones con otras variables se agruparon en cinco categorías: no lee ni escribe, primario incompleto, primario completo (incluye primario completo y secundario incompleto), secundario completo (incluye secundario completo y terciario o universitario incompleto), superior (terciario y universitario completo).

- Aspectos clínicos.

- Comorbilidades (antecedentes autorreferidos por el paciente al profesional de la salud): hipertensión arterial, eventos cardiovasculares (infarto, accidente cerebrovascular), dislipemias, esteatosis severa, hepatitis crónica o insuficiencia hepá- tica, trastornos en la coagulación o enfermedad crónica de los glóbulos rojos.

- Complicaciones crónicas (antecedentes autorreferidos al profesional de la salud): oculares, renales, neuropatías.

- Control metabólico: hemoglobina glicosilada $(\mathrm{HbA} 1 \mathrm{c})$, promedios de glucemias de los últimos tres meses según monitoreo glucémico (se consideraron los promedios de pacientes que referían complicaciones crónicas renales avanzadas, insuficiencia hepática y enfermedades hemáticas) y cumplimiento de los objetivos glucémicos. Se definió si cumplía o no con los objetivos de control glucémico o metabólico en forma individualizada. Para esto se categorizaron tres grupos según antecedentes y características como lo refirieran los estándares de la Asociación Americana de Diabetes (ADA) y la Asociación Europea para el Estudio de la Diabetes ${ }^{17}$ (AEED) (Anexo).

- Calidad de vida: ocupación y como consecuencia de la DM2 como ausentismo, jubilación prematura, reducción del sueldo por ausentismo y percepción de deterioro de calidad de vida.

Se definieron activos a aquellos encuestados que trabajaban. Se diferenciaron los encuestados: mayores de 65 años de pensionados y dentro de los jubilados los que percibieron jubilación prematura por DM y los activos (aquellos que a pesar de estar jubilados aún trabajaban), los con prestación en salud y los sin prestación en salud.

- Aspectos socioeconómicos.

- Gasto de bolsillo del paciente: se cuantificó qué proporción abarcó el gasto en salud por mes que incluyó el total en medicación para la diabetes exclusivamente, más el total en tiras reactivas, el total en medicación ajena a la diabetes, el total en gasto de consultas al médico, estudios (análisis de sangre y orina, ecografías, estudios cardiológicos, etc.) en relación con el ingreso del grupo familiar que convivía directamente con el paciente. Se analizaron si influían negativa o positivamente las variables como número de personas que viven en la familia, número de menores de edad convivientes, número de mayores de 65 años convivientes, número de jubilados prematuramente por invalidez, presencia de subsidio o ayuda familiar ajeno al grupo conviviente, presencia de mujeres en edad fértil en la familia (15-45 años).

- Hogar de pertenencia: a todos aquellos que convivían con el paciente encuestado y participaban en el consumo y la economía familiar. 
- Ingreso per cápita: cociente entre el ingreso total del grupo familiar y la cantidad de convivientes.

- Ayuda económica: presencia de subsidio otorgado por el Estado o cualquier tipo de ayuda brindada ajena al grupo familiar conviviente.

- Jefe de hogar: se refirió al conviviente que más aportaba dinero en la economía del grupo familiar.

\section{Análisis estadístico}

Se confeccionó una ficha para la recolección de datos y luego una base de datos en Excel que se procesaron con InfoStat. Se utilizaron datos expresados como media, desviación estándar, mediana y porcentajes sobre ocho regiones de Argentina y según tipo de prestación en salud. Se analizaron, según porcentuales, variables incluidas de calidad de vida: ausentismo, reducción del sueldo, jubilación prematura por DM2, percepción de pérdida de calidad de vida, percepción de subsidio, gasto de bolsillo en salud.

Se analizaron diferencias significativas por región geográfica por test de Pearson chi para jubilación prematura por DM2, percepción de calidad de vida, cumplimiento de objetivos metabólicos y por test de Kruskal Wallis para gasto de bolsillo en salud.

Se estudió el gasto del bolsillo del paciente compuesto por el total en medicación para la diabetes exclusivamente más el total en tiras reactivas, el total en medicación ajena a la diabetes, el total en gasto de consultas al médico, estudios realizados en relación con el ingreso del grupo familiar que convivía directamente con el paciente. Se analizó la influencia negativa o positiva de variables socioeconómicas como número de personas que vivían en la familia, menores de edad convivientes, mayores de 65 años convivientes, jubilados prematuramente por invalidez, recepción de subsidio o ayuda familiar ajena al grupo conviviente, presencia de mujeres en edad fértil en la familia (15-45 años de edad) y cumplimiento de objetivos metabólicos.

\section{Reparo ético}

Se presentó el protocolo base aprobado y realizado en $2013^{12}$. En los centros de reclutamiento que fue necesario se aguardó autorización de la Dirección Médica de la institución correspondiente o de los Comités de Docencia e Investigación. Se solicitó la firma del consentimiento informado en aquellos centros de reclutamiento que la institución lo exigió. Se realizó una encuesta por autorreferencia. Se preservó la confidencialidad de la información otorgada por los individuos encuestados, además de los consentimientos.

\section{RESULTADOS}

De un total de 1.520 pacientes, 802 eran mujeres y 685 hombres (no contestaron para el sexo 33 pacientes); la edad media resultó de 60,2 años y la antigüedad de DM2 referida correspondió a 10,1 años. La media de $\mathrm{HbA} 1 \mathrm{c}$ fue de $7,85 \%$ y las medias glucémicas de 150,34 mg/dl. En relación al nivel de educación alcanzado para el grupo con obra social, el mayor porcentual correspondió a terciario y universitario completo, para el grupo sin obra social al primario completo y al incompleto, en el grupo de jubilados al primario completo y el incompleto. Respecto de la prevalencia de complicaciones crónicas, los mayores porcentuales correspondieron al grupo sin obra social. Las medias de HbA1c y las medias de glucemia en los últimos tres meses más elevadas correspondieron al grupo sin obra social, por consiguiente el grupo según prestación en salud que presentó mayor porcentual de no cumplimiento de objetivos metabólicos perteneció a los sin obra social. Al analizar el cumplimiento de los objetivos metabólicos según características individuales por ADA y AEED (2012), el grupo de mayor porcentual de cumplimiento metabólico perteneció a los jubilados y los con prestación (Tabla 3).

Se estudiaron según porcentuales por región geográfica variables incluidas de calidad de vida: ausentismo y reducción del sueldo, jubilación prematura por DM, percepción de pérdida de calidad de vida, percepción de subsidio, gasto de bolsillo en salud en el año 2015 (Tabla 4). Respecto del cumplimiento de los objetivos metabólicos, las regiones con porcentuales de mayor cumplimiento de mayor a menor correspondieron a Ciudad Autónoma de Buenos Aires (CABA), Córdoba y Buenos Aires. Las regiones que presentaron mayor prevalencia en jubilación prematura por DM2 correspondieron a Córdoba, Noreste y Buenos Aires. El mayor porcentual de percepción de pérdida de calidad de vida correspondió a Noroeste, Cuyo y Litoral. Es interesante resaltar que en cuatro regiones la percepción de pérdida de calidad de vida superó el 50\%. Las regiones que recibían mayor porcentual de subsidios o ayuda económica correspondieron a Noroeste, Noreste y Comahue.

Se estudiaron, según porcentuales y tipo de prestación en salud, variables de control metabólico y aquellas incluidas de calidad de vida: ausen- 
tismo y reducción del sueldo, jubilación prematura por DM, percepción de pérdida de calidad de vida y percepción de subsidio. El grupo que refirió faltar al trabajo en mayor porcentual a causa de su diabetes correspondió a los sin obra social y por consiguiente presentaron mayor porcentual de reducción de su sueldo por ausentismo; éstos también refirieron mayor porcentual en la prevalencia de percepción de pérdida de calidad de vida. Respecto del subsidio o ayuda económica, haciendo referencia a la presencia de subsidio otorgado por el Estado o cualquier tipo de ayuda brindada ajena al grupo familiar conviviente, correspondió al grupo de jubilados, y le siguió el grupo de los sin obra social. Las diferencias fueron estadísticamente significativas para todas las variables estudiadas excepto la presencia de subsidio (p:0,066) (Tabla 5).

Al analizar las diferencias de calidad de vida por región geográfica se encontraron desigualdades significativas por región geográfica por test de Pearson chi para: jubilación prematura por DM2 ( $p: 0,000$ ), la pérdida de percepción de calidad de vida ( $p: 0,000)$, el cumplimiento de objetivos metabólicos ( $\mathrm{p}: 0,000)$ y por test de Kruskal Wallis para gasto de bolsillo en salud (p:0,000583).

Se evaluó, teniendo en cuenta que se reclutaron los datos en el primer semestre del año 2015, el gasto del bolsillo del paciente compuesto por: el total en medicación para la diabetes exclusivamente más el total en tiras reactivas, el total en medicación ajena a la diabetes, el total en gasto de consultas al médico y estudios realizados en relación al ingreso del grupo familiar que convivía directamente con el paciente (Tabla 6).
El porcentaje de gasto de bolsillo en salud en relación al total de ingreso por hogar reportado/ mes resultó una media cercana al 9\%. La relación jefe de familia fue hombre/mujer 943/470 (no contestaron 107) y la media edad jefe de familia hombre/ mujer resultó 60.04/59.26 años. El porcentual de gasto de bolsillo en salud asignado al tratamiento de la diabetes correspondió a un 52,9\%.

Se analizó la influencia negativa o positiva de variables socioeconómicas y de calidad de vida en el gasto de bolsillo como: número de personas que vivían en la familia, número de menores de edad convivientes, número de mayores de 65 años convivientes, número de jubilados prematuramente por invalidez, presencia de subsidio o ayuda familiar ajena al grupo conviviente, presencia de mujeres en edad fértil en la familia (15-45 años de edad) y el cumplimiento de objetivos metabólicos.

No se encontró correlación por Spearman del gasto de bolsillo con el número de convivientes ( $p: 0,07908)$, número de convivientes menores de 18 años ( $\mathrm{p}: 0,1934)$, los convivientes mayores de 65 años (p:0,7245) y mujeres en edad fértil (p:0,3086). Aplicando Test de Mann Whitney se encontró asociación significativa al vincular el gasto de bolsillo con la presencia de subsidio familiar o ajena al grupo conviviente $(p<0,001)$, con la jubilación prematura por DM2 $(p<0,001)$ y diferencias significativas entre los valores medianos del gasto en salud asignado por mes y el cumplimiento de objetivos metabólicos con una $p$ valor $<0,0001$; no fue así con el tipo de prestación en salud ( $p: 0,851)$.

\begin{tabular}{|l|c|c|c|}
\hline \multicolumn{1}{|c|}{ Variable } & OS & SOS & Jubilados \\
\hline Sexo: Fem/Masc & $240 / 234$ & $256 / 218$ & $306 / 233$ \\
\hline Edad: X, DS & $56,59(12,04)$ & $54,38(11,64)$ & $69,87(9,53)$ \\
\hline Antigüedad: DM2, años & $8,95(6,82)$ & $8,85(7,6)$ & $12,64(10,5)$ \\
\hline Estudios: (n, \%) & & & \\
NL & $3(0,62)$ & $13(2,68)$ & $21(3,79)$ \\
PI & $35(7,27)$ & $111(22,88)$ & $123(22,20)$ \\
PC & $99(20,58)$ & $123(25,36)$ & $189(34,11)$ \\
SI & $43(8,93)$ & $96(19,79)$ & $66(11,91)$ \\
SC & $112(23,28)$ & $82(16,9)$ & $79(14,25)$ \\
TI-UI & $40(8,31)$ & $23(4,74)$ & $13(2,34)$ \\
TC-UC & $121(25,15)$ & $16(3,29)$ & $32(5,77)$ \\
NC & $28(5,82)$ & $21(4,32)$ & $31(3,59)$ \\
\hline
\end{tabular}




\begin{tabular}{|l|c|c|c|}
\hline \multicolumn{1}{|c|}{ Variable } & OS & SOS & Jubilados \\
\hline Antecedentes: (n, \%) & & & \\
HTA & $223 / 257(53,54 \%)$ & $187 / 297(61,36 \%)$ & $181 / 373(67,32 \%)$ \\
EC & $426 / 55(11,43)$ & $420 / 65(13,30)$ & $460 / 94(16,96)$ \\
DLP & $255 / 226(46,98)$ & $237 / 248(51,13)$ & $282 / 272(49,09)$ \\
RTN & $371 / 110(22,86)$ & $285 / 200(41,23)$ & $370 / 184(33,21)$ \\
IR & $461 / 20(4,15)$ & $416 / 67(13,87)$ & $490 / 64(11,55)$ \\
Neuropatia & $416 / 65(13,51)$ & $341 / 144(42,22)$ & $433 / 121(21,84)$ \\
Alt. hepáticas & $445 / 36(7,84)$ & $429 / 56(13,05)$ & $482 / 72(14,93)$ \\
\hline HbA1c & $7,45(1,56)$ & $8.22(4,36)$ & $7,88(1,46)$ \\
Glucemias mg/dl: X, DS & $140.88(49,8)$ & $164.98(63,49)$ & $145,17(42,91)$ \\
\hline Objetivos de control Cumpl. /no cumpl: n, \% Cumplimiento metabólico según & $260 / 150(63,41)$ & $197 / 196(50,12)$ & $299 / 166(64,30)$ \\
grupos por objetivos de control ADA/AEED: (n, \%) & $167 / 64(61,67))$ & $103 / 72(58,85)$ & $141 / 45(75,80)$ \\
\hline 1 & $88 / 86(50,57)$ & $91 / 121(42,92)$ & $150 / 117(56,17)$ \\
2 & $5 / 0(100)$ & $3 / 6(33,33)$ & $8 / 4(66,66)$ \\
\hline 3
\end{tabular}

Fuente: elaboración propia.

OS: obra social; SOS: sin obra social; Fem: femenino, Masc: masculino; X: media; DS: desvío estándar; NL: no lee ni escribe; PI: primario incompleto; PC: primario completo; SI: secundario incompleto; SC: secundario completo; TI: terciario incompleto; TC: terciario completo; UI: universitario incompleto; UC: universitario completo; NC: no contestó; HTA: hipertensión arterial; EC: eventos cardiovasculares; DLP: dislipemia; RTN: complicaciones oculares; IR: complicaciones renales; Alt. hepat.: trastornos hepáticos.

Tabla 3: Antecedentes sociodemográficos y características clínicas.

\begin{tabular}{|l|c|c|c|c|c|c|c|c|}
\hline \multicolumn{1}{|c|}{ Región } & OS & SOS & JUB & $\begin{array}{c}\text { \% Cumpl. } \\
\text { objetivos }\end{array}$ & $\begin{array}{c}\% \\
\text { Jub. prem. }\end{array}$ & $\begin{array}{c}\text { \%Percep. } \\
\text { calidad de vida }\end{array}$ & $\begin{array}{c}\% \\
\text { Subs. }\end{array}$ & $\begin{array}{c}\text { Gasto de bolsillo } \\
\text { X, DS, M,IC }\end{array}$ \\
\hline Buenos Aires & 54 & 86 & 121 & $67,79 \%$ & $37,93 \%$ & $43,68 \%$ & 13,23 & $\begin{array}{c}742,8 ; 928,82 ; 500 ; \\
(200-811,1)\end{array}$ \\
\hline CABA & 63 & 62 & 42 & $80,14 \%$ & $3,01 \%$ & $34,34 \%$ & 10,24 & $\begin{array}{c}729,1 ; 589,56 ; 600 ; \\
(337,5-1000)\end{array}$ \\
\hline Comahue & 91 & 54 & 79 & $53,22 \%$ & $36,61 \%$ & $50,89 \%$ & 10,76 & $\begin{array}{c}415,1 ; 139,34 ; 484,1 \\
(350-512,5)\end{array}$ \\
\hline Córdoba & 12 & 5 & 50 & $75,00 \%$ & $74,63 \%$ & $22,39 \%$ & 9,09 & $\begin{array}{c}867 ; 1195,11 ; 500 \\
(312.8-700)\end{array}$ \\
\hline Cuyo & 83 & 79 & 67 & $60,51 \%$ & $24,12 \%$ & $53,51 \%$ & 8,41 & $\begin{array}{c}443.8 ; 595.66 ; 300 \\
(100-500)\end{array}$ \\
\hline Litoral & 58 & 43 & 87 & $43,48 \%$ & $8,1 \%$ & $53,19 \%$ & 10,64 & $\begin{array}{c}591.4 ; 455,48 ; 500 \\
(262,5-800)\end{array}$ \\
\hline NOE & 49 & 67 & 24 & $48,84 \%$ & $27,14 \%$ & $63,57 \%$ & 20,71 & $\begin{array}{c}770,5 ; 725,28 ; 500 \\
(275-925)\end{array}$ \\
\hline NE & 71 & 89 & 84 & $58,11 \%$ & $40,57 \%$ & $43,03 \%$ & 14,35 & $\begin{array}{c}513,1 ; 487,85 ; 360 \\
(200-600)\end{array}$ \\
\hline
\end{tabular}

Fuente: elaboración propia.

Cumpl. objetivos: cumplimiento de objetivos; Jub. Prem.: jubilación prematura; Percep. calidad de vida: percepción de pérdida de calidad de vida; Subs.: subsidio; X: media; DS: desvío estándar; M: mediana; IC: intervalo de confianza.

Tabla 4: Variables de calidad de vida y de objetivo metabólico según región.

\begin{tabular}{|l|c|c|c|c|}
\hline \multicolumn{1}{|c|}{ Último trimestre } & OS & SOS & Jubilados & Test Chi valor $\mathbf{p}$ \\
\hline HbA1c: X, DS & $7,45(1,56)$ & $8,22(4.36)$ & $7,88(9,37)$ & \\
\hline Medias de glucemias: X, DS & $140,88(49,8)$ & $164,98(63.49)$ & $145,17(42,91)$ & \\
\hline Falto al trabajo por DM2\% (n:1.519) & $11,23(\mathrm{n}: 481)$ & $16,49(\mathrm{n}: 485)$ & $4,34(\mathrm{n}: 553)$ & $<0,0001$ \\
\hline Redujo su sueldo por ausentismo\% (n:1.518) & $8,11(\mathrm{n}: 481)$ & $18,18(\mathrm{n}: 484)$ & $5,06(\mathrm{n}: 553)$ & $<0,0001$ \\
\hline Jubil.prematura por DM2\% (n:1.518) & $12,89(\mathrm{n}: 481)$ & $14,26(\mathrm{n}: 484)$ & $56,60(\mathrm{n}: 553)$ & $<0,0001$ \\
\hline Percp. de pérdida de calidad de vida\% (n:1.518) & $44,91(\mathrm{n}: 481)$ & $57,23(\mathrm{n}: 484)$ & $40,33(\mathrm{n}: 553)$ & $<<0,0001$ \\
\hline Percp. de subsidio\% (n:1.504) & $9,28(\mathrm{n}: 474)$ & $13,28(\mathrm{n}: 482)$ & $13,69(\mathrm{n}: 548)$ & 0,06621 \\
\hline
\end{tabular}

Fuente: elaboración propia.

X: media; DS: desvío estándar; Jubil: jubilación; Percp.: percepción.

Tabla 5: Variables de calidad de vida y de objetivo metabólico según prestación. 


\begin{tabular}{|l|c|c|c|c|c|c|}
\hline \multicolumn{1}{|c|}{ Variable } & N & Media & DS & Mínimo & Máximo & Mediana \\
\hline Gasto de bolsillo total \$ & 350 & 609,4 & 694,71 & 10 & 5000 & 400 \\
\hline Gasto medicación \$ & 199 & 417,9 & 320,05 & 0 & 2500 & 400 \\
\hline Gasto tiras reactivas \$ & 129 & $218 ., 1$ & 215,61 & 15 & 1500 & 160 \\
\hline Gasto ajeno a DM \$ & 280 & 286,5 & 447,65 & 10 & 6000 & 200 \\
\hline Gasto consultas al médico \$ & 238 & 396,8 & 717,76 & 5 & 6000 & 209.5 \\
\hline Gasto estudios médico \$ & 802 & 1643 & 2050,78 & 0 & 25000 & 1000 \\
\hline Número de convivientes \$ & 1501 & 2,68 & 1,5 & 1 & 10 & 2 \\
\hline Ingreso familiar \$ & 1062 & 8518,43 & 7903,1 & 400 & 90000 & 6000 \\
\hline Ingreso per cápita \$ & 879 & 4196 & $63 ., 4$ & 0 & 40000 & 3000 \\
\hline
\end{tabular}

Fuente: elaboración propia.

Tabla 6: Análisis sobre la composición de gastos en pesos argentinos (2015).

\section{DISCUSIÓNY CONCLUSIONES}

El impacto negativo del ausentismo, la reducción del sueldo y la percepción de pérdida de calidad de vida fueron significativamente mayores en el grupo sin prestación en salud. Además en cada región se observó disparidad entre las variables de calidad de vida, el gasto en salud y la percepción de subsidios.

A pesar de la modificación en la reglamentación nacional, el impacto negativo en el gasto de salud afecta más fuertemente a los núcleos familiares más vulnerables. Como se informó en la Encuesta Nacional de Factores de Riesgo la mayor prevalencia de DM2 se asoció al menor nivel de ingresos, educación y cobertura en salud de tipo pública ${ }^{10}$. En este estudio se reforzó este concepto pues se observó que la población sin obra social -aquellos más vulnerables, con mayor pérdida de calidad de vida, mayor disminución porcentual de su ingreso mensual a consecuencia de la DM por intercurrencias y ausentismo, los que coincidentemente tuvieron menor nivel de educación alcanzado- presentó peor control metabólico, mayores tasas de incumplimiento de objetivos glucémicos según características individuales y elevada prevalencia de complicaciones de la enfermedad.

En un trabajo sobre determinantes del gasto de bolsillo en salud e implicancias para el seguro universal en $\mathrm{México}^{18}$ se informó que la presencia de niños y/o personas de la tercera edad, familias no aseguradas sin importar su nivel de ingreso, residir en un área rural o periférica son factores que influyen significativamente y de forma positiva en el gasto de bolsillo; advirtieron también que más del 50\% del gasto total en salud era gasto privado y que más del $90 \%$ provenía directamente del bolsillo. En Estados Unidos se refirió que las personas con diabetes diagnosticada incurren en gastos médicos donde el $57,6 \%$ se atribuyó a diabetes ${ }^{19}$. En un estudio en una obra social de Argentina se observó que sobre el porcentual asignado a salud, un $60,7 \%$ por mes correspondió a tratamiento para la DM2 y que las familias más numerosas invertían menos dinero en el tratamiento de la enfermedad. En el multicéntrico el gasto asignado a DM resultó en un 52,9\%. Se infirió que el gasto de bolsillo en salud respecto del ingreso del grupo familiar mensual representó una media del $10,57 \%$, y en el multicéntrico una media del $9 \%$. No se informó si la vivienda correspondía a región rural o región perteneciente a zonas de mayor pobreza o región urbana. A su vez, en dicho estudio, al analizar variables que influyeran negativa o positivamente en el gasto de bolsillo en salud se encontró una asociación negativa entre la cantidad de personas convivientes en el hogar y el porcentaje del presupuesto invertido en la enfermedad, y una asociación positiva entre la proporción del gasto de bolsillo en salud y los respondedores con subsidio o ayuda económica ${ }^{12}$. En el multicéntrico la asociación con el número de convivientes no resultó significativa pero el valor fue muy cercano a denotarlo (p:0,079). Se advirtió una asociación significativa al relacionar el gasto de bolsillo con la presencia de subsidio o ayuda económica ajena al grupo conviviente, la jubilación prematura por DM2 y el cumplimiento de objetivos metabólicos.

Se informó que el apoyo social se relacionó con el tratamiento de la DM2. La mayoría de los pacientes con bajos niveles de apoyo social tuvo un peor estado de salud y bienestar general, y además de presentar otras comorbilidades agregadas, manifestó un $55 \%$ más de probabilidades de fallecer que aquellos con moderados niveles de apoyo social ${ }^{20}$. 
En el presente estudio sólo se consideró si los pacientes recibían ayuda económica o subsidio de algún tipo, pero no se diferenció si entre éstos estaba incluido algún tipo de apoyo social que pudiera influir no sólo en el gasto en salud sino también en variables de control metabólico y calidad de vida.

Brechas en la cobertura en salud para los pacientes con cobertura, ya sea obra social o jubilados, pueden aumentar el gasto de bolsillo al tener que pagar el precio completo de los medicamentos prescriptos y en consecuencia pueden disminuir el consumo de medicación o la adherencia al tratamiento, con la elevación del riesgo de aumentar complicaciones o eventos vasculares. Lo mismo puede inferirse para los pacientes sin obra social que pertenecen a planes nacionales o provinciales cuando la medicación que debería estar incluida en la provisión fue discontinuada en situaciones de irregularidades o crisis económica, sumado a tiempos de alta inflación. La falta de cobertura implica que queda al descubierto el consumo de medicación durante períodos significativos.

En diferentes regiones de Argentina se observó disparidad entre las variables de calidad de vida, el gasto en salud y la percepción de subsidios. La relación existente entre menores ingresos, el nivel educativo y la diabetes comprueba la necesidad de educar a la población en la promoción de la salud, la prevención y el tratamiento, con énfasis en los grupos más vulnerables. En el sistema de salud deben promoverse los recursos y métodos (inercia terapéutica) con bases en un equipo interdisciplinario que aborde de manera integral pilares terapéuticos para el logro de las metas recomendadas de tratamiento y prevención propuestas por los organismos nacionales o internacionales.

\section{Limitaciones}

Se trata de un estudio de tipo transversal que recolectó datos a través de una encuesta autorreferida con lo cual pueden haberse subestimado datos. El cuestionario interrogó sobre los resultados de control metabólico correspondientes a los últimos tres meses de realizada la encuesta. El número de pacientes encuestados en regiones como Córdoba puede haber arrojado resultados y consecuentemente conclusiones no del todo representativas. A su vez es posible el error aleatorio asociado a una muestra de tamaño limitado dado que no se contemplaron conglomerados de región detalladamente representativos. La recolección de los datos fue en el primer semestre de 2015, por lo cual a la fecha de la publicación algunas variables pueden intervenir en los resultados si se quisiera extrapolar a la actualidad dada la alta inflación de los últimos años y el consecuente impacto en los costos y gastos en salud.

\section{Agradecimientos}

En especial al Dr. Jorge Alvariñas, referente y maestro de la Nutrición y la Diabetes en Argentina y Latinoamérica, quien en el año 2001 presentó resultados sobre un estudio (que no publicó) donde informaba a través de un cuestionario sobre el cumplimiento en el tratamiento de la DM2 y su consecuente gasto de bolsillo en el Policlínico Bancario ubicado en CABA. Fue su presentación, el impacto de su disertación y una discusión de café junto con otro profesor referente en la investigación, sumado a un contexto nacional de emergencia económica, las grandes brechas en salud, las inequidades y los incumplimientos con respecto a las leyes y las reglamentaciones vigentes en ese momento que comenzó el camino en esta arista de la investigación científica relacionada con la adherencia en el tratamiento de las patologías crónicas no transmisibles, con especial énfasis en la diabetes. Arista que implicó ahondar en significados de conceptos que hacen a la adherencia al tratamiento en diabetes, la modificación del mencionado concepto según los tiempos, la historia y el uso que se le fue dando, sus diferencias con respecto al cumplimiento, la inclusión de variables cualitativas y factores relativos al concepto, resultados de investigaciones al momento y limitaciones en el tema, entre otros.

\section{BIBLIOGRAFÍA}

1. Honorable Congreso de la Nación Argentina, 1998. Ley $N^{\circ}$ 23.753. Decreto reglamentario 1271. Ley Nacional de protección al diabético. Programa de divulgación sanitaria (Internet) 1998. Citado en 2014. Disponible en: www.diabetes.org.ar/ espacio-para-la-comunidad/articulos-de-interes.

2. Honorable Congreso de la Nación Argentina (Internet) 2013. Ley 26.914. Boletín Oficial 27/12/2013. Citado en 2014. Disponible en: http://www.diabetes.org.ar/docs/Ley_26.914_modificacion_Ley_23.753.pdf.

3. Ministerio de Salud de la Nación. Resolución 201/2002. Programa Médico Obligatorio de Emergencia (PMOE) integrado por el conjunto de prestaciones básicas esenciales garantizadas por los agentes del Seguro de Salud (Internet) 2004. Citado en marzo 2015. Disponible en: http://www.sssalud.gov.ar/normativas/consulta/000595.pdf.

4. Poder Ejecutivo Nacional. Normas de provisión de medicamentos e insumos para personas con diabetes mellitus. Anexo 1 (Internet) 2018. Citado en 2018. Disponible en: http://www. cofa.org.ar/wp-content/uploads/2018/11/ANEXO-I.pdf. 
5. Gagliardino JJ. Prevención en diabetes: evidencias epidemiológicas y económicas para la toma de decisiones. Simposio 2008. Revista de la Sociedad Argentina de Diabetes 2009; 43(1):5-6.

6. Gagliardino JJ, Olivera E, Barragán H, Puppo R. A simple economic evaluation model for selecting diabetes health care strategies. Diabetic Medicine 1993; 10 (4):351-354.

7. Ministerio de Salud de la Nación. Segunda Encuesta Nacional de Factores de Riesgo para Enfermedades NoTrasmisibles. Ministerio de Salud de la Nación Argentina 2009. Disponible en: http://www.bvs.org.ar/pdf/enfr2009.pdf.

8. RozenfeldY, Hunt JS, Plauschinat C, Wong KS. Oral antidiabetic medication adherence and glycemic control in managed care. Am J Manag Care 2008; 14:71-5.

9. Bernard DM, Banthin, JS, Encinosa, WE. Health care expenditure burdens among adults with diabetes in 2001. Med Care 2006; 44(3):210-5.

10. Ministerio de Salud de la Nación. Tercera Encuesta Nacional de Factores de Riesgo, 2013. Internet 2015. Citado en 2015. Disponible en: http://www.msal.gob.ar/images/stories/bes/graficos/000 0000544cnt2015_09_04_encuesta_nacional_factores_riesgo.pdf.

11. OMS, 2004. Adherencia a los tratamientos a largo plazo: pruebas para la acción. Ginebra. Internet 2004. Citado en 2015. Disponible en: http://www.paho.org/Spanish/AD/DPC/NC/ncadherencia.html a largo plazo.

12. Linari MA. Factores que se asocian a la adherencia al tratamiento en diabetes mellitus tipo 2 en adultos del Gran Buenos Aires pertenecientes a la Unión Obrera Metalúrgica seccional, Vicente López, durante 2013. Tesis de Maestría en Epidemiología, Gestión y Políticas de Salud. Universidad Nacional de Lanús, 2015. Recuperado a partir de: http:www.unla.edu.ar/ index.php/servicios-de-la-biblioteca.

13. Alvariñas J. Adherencia al tratamiento y costos en la Sección de Nutrición del Policlínico Bancario en 2001. Documento no publicado al cuidado del autor, 2001. Sección de Nutrición del Policlínico Bancario del Gobierno de la Ciudad de Buenos Aires.
14. Linari MA, González C, Alvariñas J. Desarrollo y validación de un cuestionario sobre adherencia al tratamiento y costo de bolsillo en pacientes adultos con diabetes mellitus tipo 2. Revista de la Sociedad Argentina de Diabetes. Trabajos presentados en el XVII Congreso Argentino de Diabetes 2010; 44(4): 336-337.

15. Toobert D, Hampson S, Glasgow R. The summary of diabetes self-care activities measure: results from 7 studies and a revised scale. Diabetes Care 2000; 23(7): 943-950.

16. Gilleskie DB, Mroz TA. Estimating the effects of covariates on health expenditures. Cambridge, MA, National Bureau of Economic Research, Working Paper: 7942; 2000. Citado por: Basu A, Manning W. Issues for the next generation of health care cost analices. Medical Care 2009; 47(7), Sup.1: S109-S114.

17. ADA \& EASD 2012. Inzucchi SE, Bergenstal RM, Buse JB, Diamant M, Ferrannini E, Nauck M, Peters AL, Tsapas A, Wender R, Matthews DR; American Diabetes Association (ADA); European Association for the Study of Diabetes (EASD). Management of hyperglycemia in type 2 diabetes: a patient centered approach. Position statement of the American Diabetes Association (ADA) and the European Association for the Study of Diabetes (EASD). Diabetes Care 2012; 35(6):1364-79.

18. Torres AC, Knaul FM. Determinantes del gasto de bolsillo en salud e implicancias para el aseguramiento universal en México. Caleidoscopio de la Salud 2002; 209-225.

19. American Diabetes Association. Tesis UNLA 2013. American Diabetes Association, 2013. Economic costs of diabetes in the U.S. in 2012. Diabetes Care 2013; 36(4):1033-1046. Disponible en: www.care.diabetesjournals.org/content/36/4/1033.full.

20. Ortiz-Parada M, Baeza-Rivera MJ. Propiedades psicométricas de una escala para medir apoyo social percibido en pacientes chilenos con diabetes tipo 2. Universitas Psychologica 2011; 10(1).

\section{ANEXO}

Se categorizaron tres grupos según antecedentes y características como lo refirieran los estándares de la Asociación Americana de Diabetes y la Asociación Europea para el Estudio de la Diabetes ${ }^{17}$ :

- Grupo 1: en pacientes con DM2 de reciente diagnóstico o menos de 10 años de conocido, sin antecedentes de hipoglucemia frecuente y/o grave, sin complicaciones crónicas de la enfermedad micro o macrovasculares, $\mathrm{HbA} 1 \mathrm{c}$ de $<7 \%$ o promedios de glucemia $<150 \mathrm{mg} / \mathrm{dl}$.

- Grupo 2: en pacientes con DM2 con más de 10 años de antigüedad, antecedentes previos de hipoglucemia frecuente y/o grave, presencia de complicaciones micro o macrovasculares,
HbA1c ente $7-7,5 \%$ o promedios de glucemia entre 151-180 mg/dl.

- Grupo 3: en pacientes con DM2 con historia de hipoglucemias severas (pérdida de conocimiento o necesidad de ayuda de un segundo para el tratamiento), hipoglucemias asintomáticas, expectativa de vida limitada, condiciones comórbidas extensivas, complicaciones avanzadas (retinopatía severa, insuficiencia renal crónica establecida), analfabeto (no escribe ni lee) que haya faltado en el último trimestre a su trabajo como consecuencia de una descompensación o complicación diabética, jubilación prematura a causa de la diabetes, $\mathrm{HbA} 1 \mathrm{c}$ 7,5-8,0\% o promedios de glucemia entre 181-200 mg/dl. 